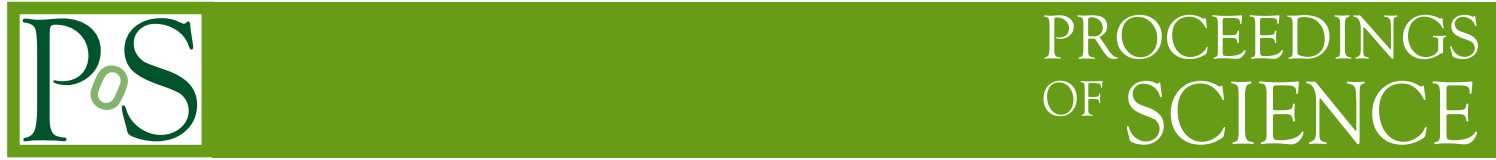

\title{
Symmetries in Non Commutative Configuration Space
}

Franciscus Vanhecke ${ }^{* \dagger}$

Univ. Federal do Rio de Janeiro (UFRJ), Brazil

E-mail: vanhecke@if.ufrj.br

\section{Antonio Roberto da Silva}

Univ. Federal do Rio de Janeiro (UFRJ), Brazil

E-mail: ardasilva@ufrj.br

\section{Cassio Sigaud Filho}

Univ. Federal do Rio de Janeiro (UFRJ), Brazil

E-mail: sigaudeif.ufrj.br

Extending earlier work [7], we examine the deformation of the canonical symplectic structure in a cotangent bundle $T^{\star}(\mathscr{Q})$ by additional terms implying the Poisson non-commutativity of both configuration and momentum variables. In this short note, we claim this can be done consistently when $\mathscr{Q}$ is a Lie group.

Fifth International Conference on Mathematical Methods in Physics 24-28, April 2006

Rio de Janeiro, Brazil

${ }^{*}$ Speaker.

${ }^{\dagger}$ Also communicated by A.R.da Silva at the First Latin American Conference on Lie Groups in Geometry, june 2006, Campinas, Brazil. 


\section{Introduction}

When a symplectic manifold is a cotangent bundle with projection, $\kappa: T^{*}(\mathscr{Q}) \rightarrow \mathscr{Q}$, and canonical symplectic structure $\omega_{0}=d q^{i} \wedge d p_{i}$, the action of a diffeomorphism $\phi$ on $\mathscr{Q}$ induces a diffeomorphism $\Phi$ on $T^{*}(\mathscr{Q})$ conserving $\omega_{0}$ :

$$
\Phi: T^{\star}(\mathscr{Q}) \rightarrow T^{\star}(\mathscr{Q}):\left\{q^{i}, p_{k}\right\} \rightarrow\left\{q^{\prime i}=\phi^{i}(q), p_{k}^{\prime}\right\} ; p_{l}=p_{k}^{\prime} \frac{\partial \phi^{k}(q)}{\partial q^{l}}
$$

In particular a group action being a homomorphism $G \rightarrow \operatorname{Diff}(\mathscr{Q})$, induces a strictly Hamiltonian action on $T^{*}(\mathscr{Q})$ :

$$
\Phi_{g}: T^{\star}(\mathscr{Q}) \rightarrow T^{\star}(\mathscr{Q}):\left(q^{i}, p_{k}\right) \rightarrow\left(q^{\prime i}=\phi^{i}(g, q), p_{k}^{\prime}\right) ; p_{l}=p_{k}^{\prime} \frac{\partial \phi^{k}(g, q)}{\partial q^{l}}
$$

Let $\mathbf{F}$ be a closed two-form on configuration space, then it is well known [1] that a change in the symplectic structure, $\omega_{0} \rightarrow \omega_{1}=\omega_{0}+\kappa^{\star} \mathbf{F}$, induces a "magnetic" interaction without changing the "free" Hamiltonian. With this new symplectic structure, the momenta variables cease to Poisson commute and one needs to introduce a potential to switch to Darboux variables.

It is then tempting to introduce also a closed two-form in the $p$-variables in such a way that Poisson non commuting $q$-variables will emerge ${ }^{1}$. In this way, we obtain a (pre-)symplectic structure :

$$
\omega=\omega_{0}-\frac{1}{2} F_{i j}(q) d q^{i} \wedge d q^{j}+\frac{1}{2} G^{k l}(p) d p_{k} \wedge d p_{l} ; d \omega=0
$$

Obviously the structure of such a two-form is not maintained by general diffeomorphisms of type (1.1). But for an affine configuration space, there is the privileged group of affine transformations, $q^{i} \rightarrow q^{\prime i}=A^{i}{ }_{j} q^{j}+b^{i}$, which conserve such a structure. When an origin is fixed, this configuration space is identified with the translation group $\mathscr{Q}=G \equiv \mathbf{R}^{N}$ with commutative Lie algebra $\mathscr{G} \equiv \mathbf{R}^{N}$ and dual $\mathscr{G}^{\star} \equiv \mathbf{R}^{* N}$. Furthermore, if $\mathbf{F}$ and $\mathbf{G}$ are constant, $\omega$ is invariant under translations. Such a situation was examined for the $\mathrm{N}$-dimensional case in our previous work [7]. From the work of Souriau and others $[1,2,4,5]$ it is clear how to generalize the first term of this extension of the canonical symplectic two-form when configuration space is a Lie group $G$ such that phase space is trivialised $T^{*} G \approx G \times \mathscr{G}^{\star}$. This is done introducing a symplectic one-cocycle, defined below.

\section{The symplectic one-cocycle}

A 1-chain $\theta$ on $\mathscr{G}$ with values in $\mathscr{G}^{\star}$, on which $\mathscr{G}$ acts with the coadjoint representation $\mathbf{k}$, $\theta \in C^{1}\left(\mathscr{G}, \mathscr{G}^{\star}, \mathbf{k}\right)$, is a linear map $\theta: \mathscr{G} \rightarrow \mathscr{G}^{\star}: \mathbf{u} \rightarrow \theta(\mathbf{u})$.

Let $\left\{\mathbf{e}_{\alpha}\right\}$ be a basis of the Lie algebra $\mathscr{G}$ with dual basis $\left\{\varepsilon^{\beta}\right\}$ of $\mathscr{G}^{\star}$ and structure constants $\left[\mathbf{e}_{\alpha}, \mathbf{e}_{\beta}\right]=\mathbf{e}_{\mu}{ }^{\mu} \mathbf{f}_{\alpha \beta}$. The 1-cochain is given by $\theta(\mathbf{u})=\theta_{\alpha, \mu} u^{\mu} \varepsilon^{\alpha}$, where $\theta_{\alpha, \mu} \doteq\left\langle\theta\left(\mathbf{e}_{\mu}\right) \mid \mathbf{e}_{\alpha}\right\rangle$.

It is a 1-cocycle, $\theta \in Z^{1}\left(\mathscr{G}, \mathscr{G}^{\star}, \mathbf{k}\right)$, if it has a vanishing coboundary:

$$
\begin{aligned}
\left(\delta_{1} \theta\right)(\mathbf{u}, \mathbf{v}) & \doteq \mathbf{k}(\mathbf{u}) \theta(\mathbf{v})-\mathbf{k}(\mathbf{v}) \theta(\mathbf{u})-\theta([\mathbf{u}, \mathbf{v}])=0 \\
\left\langle\left(\delta_{1} \theta\right)(\mathbf{u}, \mathbf{v}) \mid \mathbf{w}\right\rangle & \doteq-\langle\theta(\mathbf{v}) \mid[\mathbf{u}, \mathbf{w}]\rangle+\langle\theta(\mathbf{u}) \mid[\mathbf{v}, \mathbf{w}]\rangle-\langle\theta([\mathbf{u}, \mathbf{v}]) \mid \mathbf{w}\rangle=0 \\
\left(\delta_{1} \theta\right)_{\alpha, \mu v} & \doteq\left\langle\left(\delta_{1} \theta\right)\left(\mathbf{e}_{\mu}, \mathbf{e}_{v}\right) \mid \mathbf{e}_{\alpha}\right\rangle \\
& \doteq-\theta_{\kappa, v}{ }^{\kappa} \mathbf{f}_{\mu \alpha}+\theta_{\kappa, \mu}{ }^{\kappa} \mathbf{f}_{v \alpha}-\theta_{\kappa, \alpha}{ }^{\kappa} \mathbf{f}_{\mu v}=0
\end{aligned}
$$

\footnotetext{
${ }^{1}$ Such an approach towards non commutative coordinates was originally proposed in [6] in the two-dimensional case with posible application to anyon physics.
} 
The 1-cocycle is called symplectic if $\Theta(\mathbf{u}, \mathbf{v}) \doteq\langle\theta(\mathbf{u}) \mid \mathbf{v}\rangle$ is antisymmetric :

$$
\Theta(\mathbf{u}, \mathbf{v})=-\Theta(\mathbf{v}, \mathbf{u}) ; \Theta_{\alpha \mu} \doteq \theta_{\alpha, \mu}
$$

Any antisymmetric $\Theta$ defined in terms of $\theta \in C^{1}\left(\mathscr{G}, \mathscr{G}^{\star}, \mathbf{k}\right)$ is actually a 2 -cochain on $\mathscr{G}$ with values in $\mathbf{R}$ and trivial representation : $\Theta \in C^{2}(\mathscr{G}, \mathbf{R}, \mathbf{0})$. Furthermore, when $\theta \in Z^{1}\left(\mathscr{G}, \mathscr{G}^{\star}, \mathbf{k}\right), \Theta$ is a 2cocycle of $Z^{2}(\mathscr{G}, \mathbf{R}, \mathbf{0})$ :

$$
\begin{gathered}
\left(\delta_{2} \Theta\right)(\mathbf{u}, \mathbf{v}, \mathbf{w}) \doteq-\Theta([\mathbf{u}, \mathbf{v}], \mathbf{w})+\Theta([\mathbf{u}, \mathbf{w}], \mathbf{v})-\Theta([\mathbf{v}, \mathbf{w}], \mathbf{u})=0 \\
\left(\delta_{2} \Theta\right)\left(\mathbf{e}_{\alpha}, \mathbf{e}_{\beta}, \mathbf{e}_{\gamma}\right) \doteq-\Theta_{\kappa \gamma}{ }^{\kappa} \mathbf{f}_{\alpha \beta}+\Theta_{\kappa \beta}{ }^{\kappa} \mathbf{f}_{\alpha \gamma}-\Theta_{\kappa \alpha}{ }^{\kappa} \mathbf{f}_{\beta \gamma}=0
\end{gathered}
$$

When $\mathscr{G}$ is semisimple, $\Theta$ is exact. Indeed, the Whitehead lemmata state that $H^{1}(\mathscr{G}, \mathbf{R}, \mathbf{0})=0$ and $H^{2}(\mathscr{G}, \mathbf{R}, \mathbf{0})=0$. So, $\Theta$ is a coboundary of $B^{2}(\mathscr{G}, \mathbf{R}, \mathbf{0})$ and there exists an element $\xi$ of $C^{1}(\mathscr{G}, \mathbf{R}, \mathbf{0}) \equiv \mathscr{G}^{\star}$ such that $\Theta(\mathbf{u}, \mathbf{v})=\left(\delta_{1}(\xi)\right)(\mathbf{u}, \mathbf{v})=-\xi([\mathbf{u}, \mathbf{v}])$ or $\Theta_{\alpha \beta}=-\xi_{\mu}{ }^{\mu} \mathbf{f}_{\alpha \beta}$.

In general, $\Theta=\frac{1}{2} \Theta_{\alpha \beta} \varepsilon^{\alpha} \wedge \varepsilon^{\beta}$, with $\Theta$ obeying the cocycle condition (2.1). Acting with $L^{\star}{ }_{g^{-1} \mid g}$ : $T_{e}^{\star}(G) \rightarrow T_{g}^{\star}(G)$, yields the left-invariant forms :

$$
\begin{aligned}
& \varepsilon_{L}^{\alpha}(g) \doteq L^{\star}{ }_{g^{-1} \mid g} \varepsilon^{\alpha}=L^{\alpha}{ }_{\beta}\left(g^{-1} ; g\right) \mathbf{d} g^{\beta} \\
& \Theta_{L}(g) \doteq L^{\star}{ }_{g^{-1} \mid g} \Theta=(1 / 2) \Theta_{\alpha \beta} \varepsilon_{L}^{\alpha}(g) \wedge \varepsilon_{L}^{\beta}(g)
\end{aligned}
$$

where $L^{\alpha}{ }_{\beta}(g ; h) \doteq \partial(g h)^{\alpha} / \partial h^{\beta}$. Using the cocycle relation (2.1) and the Maurer-Cartan structure equations,

$$
\mathbf{d} \varepsilon_{L}^{\alpha}(g)=-\frac{1}{2}{ }^{\alpha} \mathbf{f}_{\mu v} \varepsilon_{L}^{\mu}(g) \wedge \varepsilon_{L}^{v}(g)
$$

it is seen that $\Theta_{L}(g)$ is a closed left-invariant two-form on $G$.

\section{G Actions on $T^{\star}(G)$}

Natural coordinates of points $x=(g, \mathbf{p}) \in T^{\star}(G)$ are given by $\left(g^{\alpha}, p_{\beta}\right)$, where $\mathbf{p}=p_{\beta} \mathbf{d} g^{\beta}$. There are two canonical trivialisations of the cotangent bundle.

- The left trivialisation :

$$
\lambda: T^{\star}(G) \rightarrow G \times \mathscr{G}^{\star}:\left(g, p_{g}\right) \rightarrow\left(g, \pi^{L}=L^{\star}{ }_{g \mid e} p_{g}=\pi_{\mu}^{L} \varepsilon^{\mu}\right)_{\mathbf{B}}
$$

which yields "body" coordinates, given by $\left(g^{\alpha}, \pi^{L} \mu\right)_{\mathbf{B}}$.

- The right trivialisation :

$$
\rho: T^{\star}(G) \rightarrow G \times \mathscr{G}^{\star}:\left(g, p_{g}\right) \rightarrow\left(g, \pi^{R}=R^{\star}{ }_{g \mid e} p_{g}=\pi_{\mu}^{R} \varepsilon^{\mu}\right)_{\mathbf{S}}
$$

which yields "space" coordinates, given by $\left(g^{\alpha}, \pi^{R} \mu\right)_{\mathbf{B}}$. 
They are related by : $\pi^{R}={R^{\star}}_{g^{-1} \mid g} \circ L^{\star}{ }_{g \mid e} \pi^{L}=\mathbf{K}(g) \pi^{L}$, where $\mathbf{K}(g)$ is the coadjoint representation of $G$ in $\mathscr{G}^{\star}$.

Lifting the left multiplication of $G$ by $G$ to the cotangent bundle yields

$$
\Phi_{a}^{L}: T^{\star}(G) \rightarrow T^{\star}(G): x=\left(g, p_{g}\right) \rightarrow y=\left(a g, p_{a g}^{\prime}=L_{a^{-1} \mid a g}^{\star} p_{g}\right)
$$

From $\lambda \circ L_{a^{-1} \mid a g}^{\star}: p_{g} \rightarrow L_{a g \mid e}^{\star} \circ L_{a^{-1} \mid a g}^{\star} p_{g}=L_{g \mid e}^{\star} p_{g}=\pi$, it is seen that, in body coordinates, $\left(\Phi_{a}^{L}\right)_{\mathbf{B}} \doteq$ $\lambda \circ \Phi_{a}^{L} \circ \lambda^{-1}:\left(g, \pi^{L}\right)_{\mathbf{B}} \rightarrow\left(a g, \pi^{L}\right)_{\mathbf{B}}$

The pull-back of the cotangent projection $\kappa: T^{\star}(G) \rightarrow G: x \doteq(g, \mathbf{p}) \rightarrow g$, yields differential forms on the cotangent bundle :

$$
\begin{aligned}
\left\langle\varepsilon_{L}^{\alpha}(x)\right| & =\kappa_{x}^{\star} \varepsilon_{L}^{\alpha}(\kappa(x)) \\
\widetilde{\Theta}_{L}(x) & =\kappa_{x}^{\star} \Theta_{L}(\kappa(x))=-\frac{1}{2} \Theta_{\alpha \beta}\left\langle\varepsilon_{L}^{\alpha}(x)\right| \wedge\left\langle\varepsilon_{L}^{\beta}(x)\right|
\end{aligned}
$$

Since $\Theta(g)$ is closed on $G$, its pull-back, $\widetilde{\Theta}_{L}(x)$, is a closed 2-form on $T^{\star}(G)$.

Furthermore, the left-invariance of $\varepsilon_{L}^{\alpha}(g): L_{a^{-1} \mid a g}^{\star} \varepsilon^{\alpha}(g)=\varepsilon^{\alpha}(a g)$ implies the $\Phi_{a}^{L}$-invariance of its pull-back : $\left(\Phi_{a}^{L}\right)_{\mid x}^{\star}\left\langle\varepsilon_{L}^{\alpha}\left(\Phi_{a}^{L}(x)\right)\right|=\left\langle\varepsilon_{L}^{\alpha}(x)\right|$ and so is $\widetilde{\Theta}_{L}(x)$. A $\Phi_{a}^{L}$-invariant basis of one-forms on $T^{\star}\left(T^{\star}(G)\right)$ is

$$
\left\{\left\langle\varepsilon_{L}^{\alpha}\right| ;\left\langle\mathbf{d} \pi^{L}{ }_{\mu}\right|\right\}
$$

The right multiplication by $a^{-1}$ induces another left action by :

$$
\Phi_{a}^{R}: T_{g}^{\star}(G) \rightarrow T_{g a^{-1}}^{\star}(G):\left(g, p_{g}\right) \rightarrow\left(g a^{-1}, p_{g a^{-1}}^{\prime}=R_{a \mid g a^{-1}}^{\star} p_{g}\right)
$$

Computing : $L_{g a^{-1} \mid e}^{\star} \circ R_{a \mid g a^{-1}}^{\star} \circ L_{g \mid e}^{\star} \pi^{L}=L_{a^{-1} \mid e}^{\star} \circ R_{a \mid a^{-1}}^{\star} \pi^{L}$, it follows that, in body coordinates, $\Phi_{a}^{R}$ acts as : $\Phi_{a}^{R}:\left(g, \pi^{L}\right)_{\mathbf{B}} \rightarrow\left(g^{\prime}=g a^{-1}, \pi^{\prime L}=\mathbf{K}(a) \pi^{L}\right)_{\mathbf{B}}$. Under $\Phi_{a}^{R}$, the $\Phi_{a}^{L}$-invariant basis (3.2) transforms as

$$
\begin{aligned}
\left(\Phi_{a}^{R}\right)_{\mid x}^{\star}\left\langle\varepsilon_{L}^{\alpha}\left(\Phi_{a}^{R}(x)\right)\right| & =\mathbf{A d}^{\alpha}{ }_{\beta}(a)\left\langle\varepsilon_{L}^{\beta}(x)\right| \\
\left(\Phi_{a}^{R}\right)_{\mid x}^{\star}\left\langle\mathbf{d} \pi^{\prime L}{ }_{\mu}\right| & =\left\langle\mathbf{d} \pi^{L}{ }_{v}\right| \mathbf{A d}^{v}{ }_{\mu}\left(a^{-1}\right)
\end{aligned}
$$

\section{The modified symplectic structure on $T^{\star}(G)$}

The canonical Liouville one-form on $T^{\star}(G)$ and its associated symplectic two-form are $\left\langle\theta_{0}\right|=$ $p_{\alpha}\left\langle d g^{\alpha}\right|=\pi_{\mu}\left\langle\varepsilon_{L}^{\mu}\right|$, and

$$
\begin{aligned}
\omega_{0} & =-\mathbf{d}\left\langle\theta_{0}\right|=-\pi_{\mu} \mathbf{d}\left\langle\varepsilon_{L}^{\mu}\right|+\left\langle\varepsilon^{\mu}\right| \wedge\left\langle\mathbf{d} \pi_{\mu}\right| \\
& =\frac{1}{2} \pi_{\mu}{ }^{\mu} \mathbf{f}_{\alpha \beta}\left\langle\varepsilon^{\alpha}\right| \wedge\left\langle\varepsilon^{\beta}\right|+\left\langle\varepsilon^{\mu}\right| \wedge\left\langle\mathbf{d} \pi_{\mu}\right|
\end{aligned}
$$

A modified symplectic two-form is obtained adding the closed two-form (3.1), constructed from the symplectic cocycle:

$$
\omega=\omega_{0}+\widetilde{\Theta}_{L}=\frac{1}{2}\left(\pi_{\mu}{ }^{\mu} \mathbf{f}_{\alpha \beta}+\Theta_{\alpha \beta}\right)\left\langle\varepsilon^{\alpha}\right| \wedge\left\langle\varepsilon^{\beta}\right|+\left\langle\varepsilon^{\mu}\right| \wedge\left\langle\mathbf{d} \pi_{\mu}\right|
$$


For semisimple $\mathscr{G}$, this reduces to :

$$
\omega=\frac{1}{2}\left(\pi_{\mu}-\xi_{\mu}\right)^{\mu} \mathbf{f}_{\alpha \beta}\left\langle\varepsilon^{\alpha}\right| \wedge\left\langle\varepsilon^{\beta}\right|+\left\langle\varepsilon^{\mu}\right| \wedge\left\langle\mathbf{d} \pi_{\mu}\right|=-\mathbf{d}\left(\left(\pi_{\mu}-\xi_{\mu}\right)\left\langle\varepsilon_{L}^{\mu}\right|\right)
$$

This means that the Liouville form is modified $\left\langle\theta_{L}\right|=\left(\left(\pi_{\mu}-\xi_{\mu}\right)\left\langle\varepsilon_{L}^{\mu}\right|\right)$ such that $\omega=-\mathbf{d}\left\langle\theta_{L}\right|$ and that $\left\{g, p_{g}^{\prime} \doteq L^{\star}{ }_{g^{-1} \mid g}(\pi-\xi)\right\}$ and there are global Darboux coordinates : $\left\{g^{\alpha}, p^{\prime}{ }_{\mu}=p_{\mu}-\right.$ $\left.\xi_{\beta} L_{\mu}^{\beta}\left(g^{-1} ; g\right)\right\}$.

Finally we may add another left-invariant and closed two-form in the $\pi$ variables $\widetilde{\Upsilon}_{L}=(1 / 2) \Upsilon^{\mu v}\left\langle\mathbf{d} \pi_{\mu}\right| \wedge$ $\left\langle\mathbf{d} \pi_{v}\right|$ such that

$$
\omega_{L}=\omega_{0}+\widetilde{\Theta}_{L}+\widetilde{\Upsilon}_{L}
$$

defines a $\Phi_{a}^{L}$-invariant (pre)-symplectic two form on $T^{\star}(G)$.

Under $\Phi_{a}^{R}$, this (pre-)symplectic two-form (4.5) is invariant if $a$ belongs to the intersection of the isotropy groups of $\widetilde{\Theta}_{L}$ and $\widetilde{\Upsilon}_{L}$ :

$$
\Theta_{\alpha \beta} \mathbf{A d}_{\mu}^{\alpha}(a) \mathbf{A d}_{v}^{\beta}(a)=\Theta_{\mu v} ; \mathbf{A d}_{\mu}^{\alpha}\left(a^{-1}\right) \mathbf{A d}_{v}^{\beta}\left(a^{-1}\right) \Upsilon^{\mu v}=\Upsilon^{\alpha \beta}
$$

\section{Conclusions}

The degeneracy of the two-form (4.4) will be examined in further work, as was done in [7] for the abelian group. If $\omega_{L}$ is not degenerate, Poisson Brackets can be defined and, in the degenerate case, the constrained formalism of [3] is applicable. Finally, if the isotropy group of (4.5) is not empty, the remaining $\Phi_{a}^{R}$-invariance will provide momentum mappings. Equations of motion of the Euler type will follow from a Hamiltonian of the form

$$
H \doteq \frac{1}{2} \mathscr{I}^{\mu v} \pi_{\mu}^{L} \pi^{L}{ }_{v}
$$

The momenta mentionned above will be conserved if the isotropy group above also conserves the inertia tensor $\mathscr{I}$.

\section{References}

[1] J-M. Souriau, Structure des systèmes dynamiques,Dunod,1970.

[2] R. Abraham and J.E. Marsden, Foundations of Mechanics,Benjamin,1978

[3] M.J. Gotay, J.M. Nester and G. Hinds, J.Math.Phys.19,2388(1978).

[4] P.Liberman and Ch-M.Marle, Symplectic Geometry and Analytical Mechanics, D.Reidel Pub.Comp.,1987

[5] J.A. de Azcárraga and J.M.Izquierdo, Lie groups, Lie algebras, cohomology and some applications in physics, Cambridge Univ.Press, 1998. 
[6] P.A. Horváthy, Ann.Phys.299,128(2002)

[7] F.J.Vanhecke, C.Sigaud and A.R.da Silva, arXiv:math-phys/0502003(2005) and Braz.J.Phys.36,no IB,194(2006) 